\title{
Calculation of cost-optimal combination of building materials and technical systems for nZEB single-family house using global cost calculation method
}

\author{
Staṇislavs Gendelis ${ }^{1, *}$, Mikus Miḳelsons ${ }^{2}$, Andris Jakovičs ${ }^{1}$, and Edgars Bredovskis \\ ${ }^{1}$ The Faculty of Physics, Mathematics and Optometry, University of Latvia, Latvia \\ ${ }^{2}$ Department of Infrastructure, University of Latvia, Latvia
}

\begin{abstract}
As a rule, during the design stage of a single-family house, only initial costs are taken into account and optimised. Long-term financial calculations are usually not even made. At the same time, total costs to design, build and operate the building show a real difference in expenses while living in this house. In the case of nearly zero energy buildings (nZEBs), this global cost approach becomes especially useful because of the strict requirements of heating and primary energy, as well as the compulsory use of renewable energy sources, requiring more detailed and comprehensive calculations of a cost-optimal set of solutions. In this study, a nZEB single-family house is analysed in detail using a comparative global cost calculation methodology. Different insulation materials and construction types, as well as two types of windows and various heating systems, are combined to obtain the same calculated annual energy consumption. The economic calculations of each combination are carried out for the calculation period of 30 years, taking into account expected energy price increase, discount rate, etc. These calculations show the costoptimal variants, allowing the prediction of a more advantageous selection of materials and system use in a nZEB in terms of the real operation costs.
\end{abstract}

\section{Introduction}

The building and construction sectors are responsible for $36 \%$ of global final energy use and $39 \%$ of energy-related $\mathrm{CO}_{2}$ emissions [1]. The EU directive on energy performance of buildings (EPDB) [2] requires member states to set minimum requirements for energy performance of buildings and elements, including new and existing buildings. This directive demands the calculation of the cost-optimal balance between the primary energy and the total cost during the lifetime of a building through the use of a comparative methodology [3]. According to this directive, all the member states have to update and revise the national requirements every five years $[4,5]$. The same methodology can also be successfully used for purposes that are more practical, such as, for example, helping to choose the most cost-effective construction option for a building.

* Corresponding author: stanislavs.gendelis@1u.1v 
Almost always, only the initial construction costs are taken into account during the design stage of a single-family house. These are also discussed and optimised with the customer with the aim of reducing them. At the same time, not only design and build costs, but also the building's operating and maintenance costs show a real difference in total expenses while living in the house in estimations of the so-called 'cost of ownership' [6,7]. Practically speaking, the cheapest building rarely results in the lowest operation costs; in fact, it is generally the other way around. In the case of nZEB buildings (mandatory requirements in Latvia from 2021), this global cost approach becomes especially useful because of the strict requirements in terms of heating and primary energy, as well as the compulsory use of renewable energy sources, which requires more detailed and more comprehensive calculations of a cost-optimal set of solutions.

In this study, a newly built nZEB single-family house (Fig. 1) is analysed in detail through using a comparative global cost calculation methodology. Different insulation materials and construction types, as well as two types of windows and various heating systems, are combined to obtain the same calculated annual energy consumption. The economic calculations of each combination are carried out for the lifetime period of 30 years, taking into account expected energy price increase, discount rate, etc. These calculations show the differences in the total cost of ownership, which allows for choosing more advantageous materials and systems during the planning phase, thus eliminating unexpected expenses later.
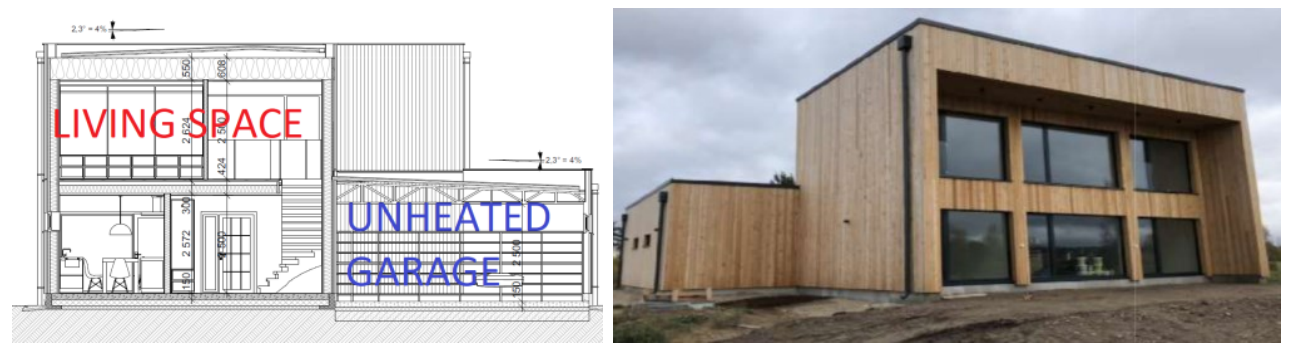

Fig. 1. Cross-section of an analysed building (left) and a photo after the construction (right).

\section{Assumptions and methods}

It is assumed that total cost of ownership consists of an initial investment (generally construction costs) and the follow-on costs (e.g. energy, utilities, cleaning, maintenance, replacement costs, etc.). The costing process includes two calculation types - energy and economic - which are described in detail below.

Thermal transmittance ( $U$-value) for building structures, as well as the information related to the ventilation system with heat recovery, provides information about heat losses during the heating season, which is dominant in Latvia due to its climate. With taking into account the climatic data together with the internal and solar heat gains, it is possible to calculate the total heating consumption of a whole building based on the EN ISO 52016-1 standard [8]. The simplified monthly calculation principle is applied for this methodology, while it also includes a calculation of the primary energy and $\mathrm{CO}_{2}$ emissions according to Latvian legislation [9]. The thickness of the used insulation materials for different constructions is slightly altered to obtain the same $U$-value, meaning this does not change the annual energy consumption. The planned $U$-values for the boundary structures are very low (see Table 2), meaning that in the case of masonry walls, a rendered facade cannot be used due to the limitation of the insulation material thickness (maximum $20 \mathrm{~cm}$ ) - thus only hinged ventilated facades are considered. Parallel to the constructions, the heating systems 
with different energy sources and efficiency are also varied, which is easy to do in the case of a new building. General building parameters are presented in Table 1, while a summary of the analysed building constructions and the different heating systems are presented in tables 2 and 3.

The second and largest calculation part consists of financial calculations (macroeconomic calculations may be made as well, taking into account $\mathrm{CO}_{2}$ costs, no taxes, modest price forecast changes, etc.) and a combination of variants (Table 4). These calculations have been made according to the chosen methodology [3], taking into account the variables summarised in Table 5. The differences in cost-optimal energy efficiency packages (combinations of measures) may vary significantly in macro-economic and financial calculations (without any improvements) since macro-economic calculations show more positive values (meaning more cost-effective investments) than with the financial calculation approach.

Several expenses were not included in the calculation, including plumbing, floor finish, inner doors, etc., because they are not directly related to energy efficiency, while inner wall, ceiling and floor base expenses were taken into account, because they are directly related to the building's main structure of masonry (clay/concrete blocks) or wooden frame.

Table 1. General nZEB building parameters.

\begin{tabular}{|c|c|c|c|c|c|c|c|c|}
\hline \multirow{2}{*}{$\begin{array}{c}\text { Floor } \\
\text { area }\end{array}$} & \multirow{2}{*}{ Volume } & \multirow{2}{*}{$\begin{array}{c}\text { Outer } \\
\text { walls }\end{array}$} & \multirow{2}{*}{$\begin{array}{c}\text { Ground/ } \\
\text { roof }\end{array}$} & & \multicolumn{6}{|c|}{ Energies, $\mathrm{kWh} / \mathrm{m}^{2}$} \\
\hline $154 \mathrm{~m}^{2}$ & $384 \mathrm{~m}^{3}$ & $214 \mathrm{~m}^{2}$ & $101 \mathrm{~m}^{2}$ & 20,9 & 0,6 & 2,1 & 3,6 & 2,7 \\
\hline
\end{tabular}

Table 2. Summary of analysed building constructions.

\begin{tabular}{|c|c|c|c|c|}
\hline Element & $\begin{array}{c}U \text {-value } \\
\left(\mathrm{W} / \mathrm{m}^{2} / \mathrm{K}\right)\end{array}$ & Main construction & Insulation material & Designation \\
\hline \multirow{8}{*}{ Outer wall } & \multirow{8}{*}{0.10} & Wooden frame & Mineral wool, $30 \mathrm{~cm}$ & $\mathrm{O} 0 \mathrm{mw}$ \\
\hline & & Wooden frame & Loose-fill cellulose, $45 \mathrm{~cm}$ & O1cel \\
\hline & & \multirow{2}{*}{ Expanded clay blocks } & Hinged ventilated facade, mineral wool, $35 \mathrm{~cm}$ & $\mathrm{O} 2 \mathrm{mw}$ \\
\hline & & & Hinged ventilated facade, EPS, $30 \mathrm{~cm}$ & O2eps \\
\hline & & \multirow{2}{*}{ Perforated concrete blocks } & Hinged ventilated facade, mineral wool, $34 \mathrm{~cm}$ & $\mathrm{O} 3 \mathrm{mw}$ \\
\hline & & & Hinged ventilated facade, EPS, $28 \mathrm{~cm}$ & O3eps \\
\hline & & \multirow{2}{*}{ Aerated concrete blocks } & mineral wool, $30 \mathrm{~cm}$ & $\mathrm{O} 4 \mathrm{mw}$ \\
\hline & & & EPS, $24 \mathrm{~cm}$ & O4eps \\
\hline \multirow{4}{*}{$\begin{array}{c}\text { Floor } \\
\text { construction }\end{array}$} & \multirow{4}{*}{0.13} & Slab-on-ground foundation & XPS, $28 \mathrm{~cm}$ & F0xps \\
\hline & & Slab-on-ground foundation & mineral wool, $28 \mathrm{~cm}$ & F1mw \\
\hline & & Slab-on-ground foundation & EPS, $27 \mathrm{~cm}$ & F2eps \\
\hline & & Strip foundation & $\mathrm{XPS}, 20 \mathrm{~cm}$ & F3xps \\
\hline \multirow{3}{*}{$\begin{array}{c}\text { Roof } \\
\text { construction }\end{array}$} & \multirow{3}{*}{0.06} & Flat roof & glass wool, $65 \mathrm{~cm}$ & R0gw \\
\hline & & Flat roof & loose-fill cellulose $65 \mathrm{~cm}$ & R1cel \\
\hline & & Flat roof & mineral wool, $60 \mathrm{~cm}$ & $\mathrm{R} 2 \mathrm{mw}$ \\
\hline \multirow{2}{*}{ Windows } & \multirow{2}{*}{0.77} & Triple glazed, wood frame & \multirow{2}{*}{$\mathrm{N}_{2}$} & W0w \\
\hline & & Triple glazed, PVC frame & & W1pvc \\
\hline
\end{tabular}

Table 3. Summary of analysed heating systems.

\begin{tabular}{|c|c|c|c|c|}
\hline Type & Designation & $\begin{array}{c}\text { SCoP (for heating only) } \\
\text { / efficiency }\end{array}$ & $\begin{array}{c}\text { Primary energy } \\
\text { factor [9] }\end{array}$ & $\begin{array}{c}\mathrm{CO}_{2} \text { factor } \\
\text { (kg/MWh) [9] }\end{array}$ \\
\hline Heat pump (borehole-water) & $\mathrm{H}$ hhpV & $3.9(5.5)$ & 1.5 & 109 \\
\hline Heat pump (earth-water) & $\mathrm{H} 1 \mathrm{hpH}$ & $4.1(5.8)$ & 1.5 & 109 \\
\hline Heat pump (air-water) & $\mathrm{H} 2 \mathrm{hpA}$ & $3.7(3.9)$ & 1.5 & 109 \\
\hline Natural gas boiler & $\mathrm{H} 3$ gas & 0.95 & 1.1 & 202 \\
\hline Pellet boiler & $\mathrm{H} 4 \mathrm{pel}$ & 0.9 & 0.2 & 0 \\
\hline Electric heaters & $\mathrm{H} 5 \mathrm{ele}$ & 1 & 1.5 & 109 \\
\hline
\end{tabular}


Table 4. Combination of construction and systems analysed (designations see in Tables 2, 3).

\begin{tabular}{|c|c|c|c|}
\hline $\begin{array}{l}\text { Combination } \\
\text { (changes are underlined) }\end{array}$ & Short description & $\begin{array}{l}\text { Initial } \\
\text { investment } \\
\left(€ / \mathrm{m}^{2}\right)\end{array}$ & $\begin{array}{l}\text { Global } \\
\text { costs } \\
\left(€ / \mathrm{m}^{2}\right)\end{array}$ \\
\hline O0mw.W0w. R0gw.F0xps.H0hpV & Original '0' variant as built (Fig. 1, right) & 1030 & 1768 \\
\hline Ocel.W0w. R0gw.F0xps.H0hpV & Changed wall and floor constructions & 1027 & 1766 \\
\hline Omw.W0w. R0gw.F3xps.H0hpV & Changed wall and floor constructions & 1345 & 2061 \\
\hline O2eps.W0w. R0gw.F3xps.H0hpV & Changed wall and floor constructions & 1314 & 2030 \\
\hline O3mw.W0w. R0gw.F3xps.H0hpV & Changed wall and floor constructions & 1349 & 2066 \\
\hline O3eps.W0w. R0gw.F3xps.H0hpV & Changed wall and floor constructions & 1306 & 2023 \\
\hline O4mw.W0w. R0gw.F3xps.H0hpV & Changed wall and floor constructions & 1342 & 2062 \\
\hline O4eps.W0w. R0gw.F3xps.H0hpV & Changed wall and floor constructions & 1311 & 2031 \\
\hline O0mw.W1pvc.R0gw.F0xps.H0hpV & Changed windows & 998 & 1720 \\
\hline O0mw.W0w. R1cel.F0xps.H0hpV & Changed roof constru & 1030 & 1772 \\
\hline O0mw.W0w. R2mw.F0xps.H0hpV & Changed roof construction & 1060 & 1806 \\
\hline O0mw.W0w. R0gw.F1mw.H0hpV & Changed floor construction & 1034 & 1773 \\
\hline O0mw.W0w. R0gw.F2eps.H0hpV & Changed floor construction & 1029 & 1767 \\
\hline O0mw.W0w. R0gw.F0xps.H1hpH & Different heating system & 1046 & 1856 \\
\hline O0mw.W0w. R0gw.F0xps.H2hpA & Different heating system & 1060 & 1932 \\
\hline O0mw.W0w. R0gw.F0xps. & Different heating system & 1053 & 1649 \\
\hline O0mw.W0w. R0gw.F0xps.H4pel & Different heating system & 1042 & 1702 \\
\hline O0mw.W0w. R0gw.F0xps.H5ele & Different heating system & 976 & 1462 \\
\hline O0mw.W0w. R2mw.F1mw.H0hpV & Mineral & 1064 & 1811 \\
\hline O2mw.W0w. R2mw.F3xps.H0hpV & Mineral wool as insulation & 1376 & 2099 \\
\hline O3mw.W0w. R2mw.F3xps.H0hpV & Mineral wool as insulation (exc. floor) & 1380 & 2104 \\
\hline O4mw.W0w. R2mw.F3xps.H0hpV & Mineral wool as insulation (exc. floor) & 1373 & 2100 \\
\hline O0mw.W1pvc.R2mw.F1mw.H0hpV & Mineral wool as + PVC windows & 1033 & 1763 \\
\hline O2mw.W1pvc.R2mw.F3xps.H0hpV & Mineral wool as insulation (exc. floor) + PVC windows & 1344 & 2051 \\
\hline O3mw.W1pvc. $\mathrm{R} 2 \mathrm{mw} . \mathrm{F} 3 \mathrm{xps} . \mathrm{H} 0 \mathrm{hpV}$ & Mineral wool as insulation (exc. floor) + PVC windows & 1348 & 2056 \\
\hline O4mw.W1pvc.R2mw.F3xps.H0hpV & Mineral wool as insulation (exc. floor) + PVC windows & 1341 & 2052 \\
\hline O1cel.W0w. R1cel.F0xps.H0hpV & Loose-fill cellulose as insulation & 1030 & 1772 \\
\hline O1cel.W1pvc.R1cel.F0xps.H0hpV & Loose-fill cellulose as insulation + PVC win & 996 & 1722 \\
\hline O2eps.W0w. R0gw.F3xps.H0hpV & EPS/XPS as insulation for walls, floor & 1314 & 2030 \\
\hline O3eps.W0w. R0gw.F3xps.H0hpV & EPS/XPS as insulation for walls, floor & 1306 & 2023 \\
\hline O4eps.W0w. R0gw.F3xps.H0hpV & EPS/XPS as insulation for walls, floor & 1311 & 2031 \\
\hline O2eps.W1pvc.R0gw.F3xps.H0hpV & EPS/XPS as insulation for walls, floor + PVC windows & 1282 & 1982 \\
\hline O3eps.W1pvc.R0gw.F3xps.H0hpV & EPS/XPS as insulation for walls, floor + PVC windows & 1275 & 1975 \\
\hline O4eps.W1pvc.R0gw.F3xps.H0hpV & EPS/XPS as insulation for walls, floor + PVC windows & 1279 & 1983 \\
\hline
\end{tabular}

Table 5. Parameters used in economic calculations.

\begin{tabular}{|l|c|c|}
\hline \multicolumn{1}{|c|}{ Parameter } & Value & Estimated lifetime, years \\
\hline Discount rate, $\%$ & $5 \%$ & \\
\hline Changes in commodity prices, $\%$ annum & $1 \%$ & \\
\hline Changes in maintenance and operating costs, $\%$ annum & $1 \%$ & \\
\hline Changes in energy prices, $\%$ annum & $1 \%$ & \\
\hline Price of energy - electricity, euro/MWh & $€ 150$ & \\
\hline Price of energy - natural gas, euro/MWh $/ \mathrm{M}$ - & $€ 41$ & \\
\hline Price of energy - wood pellets, euro/MWh & $€ 35$ & \\
\hline Maintenance cost - windows/doors, $\%$ annum & $2 \%$ & 20 \\
\hline Maintenance cost - hinged ventilated facade, $\%$ annum & $1 \%$ & 30 \\
\hline Maintenance cost - roof insulation, $\%$ annum & $2 \%$ & 30 \\
\hline Maintenance cost - slab insulation, $\%$ annum & $4 \%$ & 25 \\
\hline Maintenance cost - heat pump, $\%$ annum & $7 \%$ & 15 \\
\hline Maintenance cost - natural gas boiler, $\%$ annum & $3 \%$ & 15 \\
\hline Maintenance cost - pellet boiler, $\%$ annum & $5 \%$ & 15 \\
\hline Maintenance cost - electric heaters, $\%$ annum & $3 \%$ & 30 \\
\hline Maintenance cost - recuperation system, \% annum & $8 \%$ & 15 \\
\hline Maintenance cost - wooden frame construction & & 40 \\
\hline Maintenance cost - masonry construction & & 50 \\
\hline
\end{tabular}




\section{Results}

As the heating energy demand (MWh) for all the considered combinations is the same, only initial and global costs $(€)$ are variable and they can be analysed according to different aspects. A summary of the initial and global costs for all the combinations excluding changes in heating system is presented in Table 4 and is visually shown in Fig. 2 (where the combinations are arranged from smallest to highest initial costs). A comparison of both costs over a 30-year period clearly shows that buildings with lower construction costs also have lower global costs, while for cases with variable heating consumption, this correlation would not be so strong. The two combinations with the lowest costs are the original variant with altered PVC windows only and the combination with loose-fill cellulose used as insulation for the walls and roof construction and with PVC windows. The reason for the choice of more expensive wooden windows is generally linked to psychological and ecological aspects. The original variant is the fifth cheapest, while the difference between it and the third variant is negligible.

A comparison of the sum of initial costs and the calculated heating costs for a 30-year period for the five most popular heating systems (Table 3) is shown in Fig. 3. Here, it is clear that the most cost-effective system is the geothermal heat pump with a borehole heat exchanger. However, it is important to note that the calculations of thermal energy produced by heat pumps is highly dependent on their assumed seasonal coefficient of performance, which varies for different soil types and groundwater levels. The very low initial costs of electric heaters are not optimal in the long run due to high electricity prices, but this type of heating is economically justifiable in the shorter run.

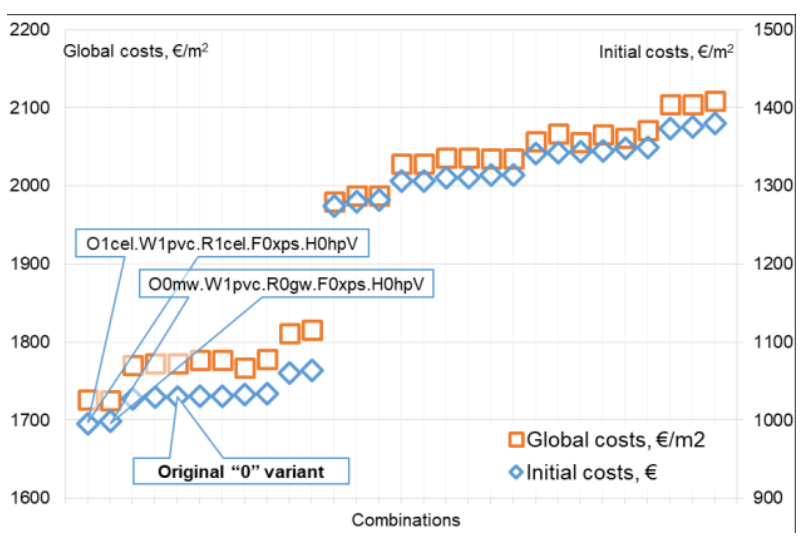

Fig. 2. Comparison of initial and global costs (see Table 5).

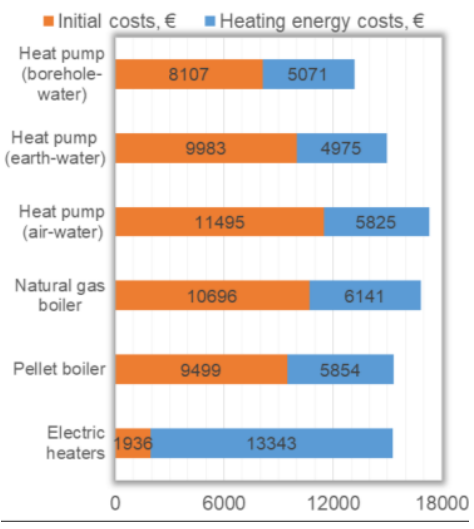

Fig. 3. Total initial and energy costs for heating system in 30 years.

\section{Conclusions}

In the case of a nearly zero energy building, due to its low energy consumption and higher building costs, long-term economic calculations using the global cost methodology are very useful to justifying the choice of materials, construction types and systems based on the real, long-term operation costs.

The most critical assumptions used in the calculation methodology, which can have a significant impact on the reliability of the results, are the forecast of future energy prices and operating and maintaining costs as well as the highly fluctuating construction costs. The choice of heating systems for the building is limited by the availability of resources and 
the possible restrictions from the local municipalities (e.g. whether it is possible to join the district heating systems; there may be ban on making an independent system).

In cases with the same heating energy consumption, initially cheaper buildings equate to lower global costs during the analysed 30-year operating period, while here, other non energy-related factors influence the customer's choice of materials and construction. In addition to the analysed ventilated facades, there is another very popular type of wall rendered facades. However, due to their limitation in terms of thermal insulation material thickness (and, therefore, lower $U$-values) this construction type is not included in the reviewed solutions. In future studies, rendered facades should be included for more objective and comprehensive market studies.

This work was supported by the European Regional Development Fund project, 'Development, optimization and sustainability evaluation of smart solutions for nearly zero energy buildings in real climate conditions' [1.1.1.1/16/A/192].

\section{References}

1. UN Environment and International Energy Agency, Towards a zero-emission, efficient, and resilient buildings and construction sector. Global Status Report 2017 (2017)

2. European Parliament and of the Council, Directive 2010/31/EU of the European Parliament and the Council on the energy performance of buildings. Official Journal of the European Union, 153, 13-35 (2010)

3. European Parliament and of the Council, Commission Delegated Regulation (EU) No. 244/2012 of 16 January 2012 supplementing Directive 2010/31/EU of the European Parliament and of the Council on the energy performance of buildings by establishing a comparative methodology framework for calculating cost-optimal levels of minimum energy performance requirements for buildings and building elements. Official Journal of the European Union, 81, 129-147 (2012)

4. European Commission, Progress by member states in reaching cost-optimal levels of minimum energy performance requirements, https:/lec.europa.eu/transparency/regdoc/ rep/1/2016/EN/1-2016-464-EN-F1-1.PDF (2016)

5. J. Karásek, J. Pojar, L. Kaločai, R. Schneiderová Heralová, Cost optimum calculation of energy efficiency measures in the Czech Republic. Energy Policy, 123, 155-166 (2018)

6. U. Bogenstätter, Prediction and optimization of life-cycle costs in early design. Build. Res. Inf., 28, 376-386 (2000)

7. D. Langdon, Life cycle costing as a contribution to sustainable construction: a common methodology - Guidance on the use of the LCC - methodology and its application in public procurement, http://ec.europa.eu/DocsRoom/documents/5060 (2007)

8. EN ISO 52016-1:2017, Energy performance of buildings - Energy needs for heating and cooling, internal temperatures and sensible and latent heat loads - Part 1: calculation procedures (2017)

9. Latvian Cabinet of Ministers, Cabinet Regulation No. 348, Regulations Regarding the Methodology for Calculating the Energy Performance of Buildings (2013) 